SISTEMA
ELETRONICO
DE REVISTAS
SER I UfPR

\title{
A defasagem de conhecimento na prática na Avaliação de Impacto Ambiental em projetos de energia eólica
}

\section{The knowledge gap in Environmental Impact Assessment practice for wind energy projects}

\author{
Izabella de Camargo AVERSA ${ }^{1 *}$, Marcelo MONTAÑO ${ }^{1}$
}

${ }^{1}$ Programa de Pós-Graduação em Ciências da Engenharia Ambiental (PPG-SEA), Escola de Engenharia de São Carlos, Universidade de São Paulo (USP), São Carlos, SP, Brasil.

*E-mail de contato: iza.aversa@gmail.com

Artigo recebido em 19 de novembro de 2018, versão final aceita em 27 de agosto de 2019.

RESUMO: A literatura tem reconhecido a importância de evidências cientificamente construídas no suporte à definição de políticas ambientais e tomada de decisão. Porém, na prática, esta interface apresenta limitações e um razoável distanciamento da teoria. A Avaliação de Impacto Ambiental (AIA) se destaca como um instrumento de planejamento e prevenção de impactos, que deve ser pautado em evidências, construídas por meio das melhores técnicas científicas, para fornecer informações suficientes e em tempo hábil para a tomada de decisão. Os mecanismos de aproximação entre o conhecimento científico sobre os potenciais impactos de uma determinada tipologia de projeto e as informações abordadas na prática da AIA constituem aspectos relativamente desconhecidos, devendo ser investigados de modo que seja possível estabelecer estratégias adequadas para esta aproximação. O presente trabalho adota como objeto de estudo a prática da AIA no setor de geração de energia eólica no Brasil, buscando identificar a distância entre o conhecimento científico acerca dos potenciais impactos desta tipologia, e aquilo que é efetivamente apresentado ao processo decisório por meio da AIA. Para tanto, foi realizada uma síntese dos impactos potenciais de projetos de geração de energia eólica, descritos na literatura internacional, e a consolidação em uma lista de verificação, a qual foi aplicada ao conteúdo de 31 Estudos de Impacto Ambiental elaborados (2010-2017) para o estado do Ceará. A partir dessa análise, foi possível identificar que, de modo geral, existe um significativo distanciamento entre a prática da AIA e o conhecimento científico. Tais resultados sugerem que as decisões a respeito da aceitabilidade dos impactos não estão sendo tomadas com base nas melhores informações disponíveis e, portanto, possivelmente não asseguram a avaliação dos impactos significativos. Por outro lado, a identificação de casos positivos pontuais destaca uma perspectiva favorável aos órgãos ambientais de atuarem na promoção da aproximação entre o estado-da-arte do conhecimento e a tomada de decisão amparada pela AIA. 
Palavras-chave: avaliação de impacto ambiental; estudo de impacto ambiental; conhecimento científico; energia eólica.

ABSTRACT: The literature has recognized the importance of scientific evidence to support environmental policies and the decision-making process. However, the practice shows limitations in this interface and a reasonable distance from theory. The Environmental Impact Assessment (EIA) stands out as an instrument for planning and impact prevention, which should be based on evidence developed through the application of the best scientific techniques to provide sufficient information and in a timely manner for the decision. The mechanisms of approximation between scientific knowledge regarding the potential impacts caused by a specific project typology and the information addressed in the practice of EIA are relatively unknown aspects. These mechanisms should be investigated in order to stablish adequate strategies for this approach. The present work aimed to identify the distance between scientific knowledge about the impacts potentially caused by wind energy projects and what is effectively presented to the decision-making process in EIA reports. The study object of this research was the EIA practice in the sector of wind energy generation in Brazil. The potential impacts of wind power generation described in the international literature were consolidated in a checklist, which was applied to the content of 31 EIA reports elaborated for projects in the state of Ceará (2010-2017), Brazil. Based on the checklist application it was possible to identify that, in general, there is a significant distance between the practice of EIA and current scientific knowledge. These results suggest that decisions about the acceptability of potential impacts are not being made based on the best available information and therefore possibly do not ensure the evaluation of significant impacts. On the other hand, the identification of specific positive cases highlights a favorable perspective for the environmental agencies to act in order to promote the approximation between the state-of-the-art of knowledge and the decision-making process supported by the EIA.

Keywords: environmental impact assessment; EIA reports; scientific knowledge; wind energy.

\section{Introdução}

A precaução é um dos mais importantes princípios que amparam a implementação de políticas ambientais e configura também um desafio, uma vez que implica em assumir limitações e incertezas na condução dos projetos de desenvolvimento (Wynne, 1992). Neste contexto, destaca-se a Avaliação de Impacto Ambiental (AIA) e a sua preocupação justamente em identificar e avaliar as consequências ambientais de uma atividade de modo prévio à realização de qualquer ação (Morrison-Saunders, 2011).

A AIA deve aplicar as melhores metodologias e técnicas científicas disponíveis e praticáveis para fornecer informações suficientes e confiáveis para a tomada de decisão, as quais devem ser focadas nos fatores-chave e nos efeitos ambientais significativos (IAIA - International Association For Impact Assessment, 1999).

A interface entre a ciência e a definição de políticas ambientais e tomada de decisão é reconhecida na literatura como algo necessário e relevante (Cortner, 2000; Holmes \& Clark, 2008; Dunn et al., 2018). Contudo, também se reconhece a existência de um distanciamento entre teoria e prática (Holmes \& Clark, 2008). Ainda que os questionamentos, modelos e estudos sobre a necessidade de aprimoramento desta interface sejam diversos, a importância da ciência para a produção de evidências científicas para informar o processo decisório tem sido amplamente destacada (Cabinet Office, 1999; Rose, 2015; Dunn et al., 2018). 
Assim como os estudos ambientais devem ser pautados em evidências científicas (IAIA, 1999), o monitoramento durante todas as etapas do projeto, desenvolvidos no âmbito da AIA, pode auxiliar a reduzir lacunas de conhecimento e incertezas em relação aos potenciais impactos e fornecer evidências científicas para a gestão do ambiente e do território (Zwart et al., 2015).

Os mecanismos de aproximação entre o conhecimento científico e as informações efetivamente empregadas em processos de tomada de decisão constituem aspectos que carecem de esclarecimento. Desse modo, o presente artigo visa identificar se há distanciamento entre o estado-da-arte do conhecimento sobre os impactos potenciais de uma atividade e aquilo que é efetivamente informado aos tomadores de decisão por meio da AIA. O objeto de estudo da pesquisa foi o sistema de AIA e licenciamento ambiental para projetos de geração de energia eólica do estado do Ceará.

A escolha do setor de energia eólica é justificada por constituir uma tipologia de projeto relativamente nova no Brasil e, portanto, permitir estabelecer com segurança qual seria esta distância. Além disso, o fato da energia eólica ser considerada como não poluente, devido a não gerar emissões atmosféricas ou resíduos durante a operação (Leung \& Yang, 2012; Mirasgedis et al., 2014), cria uma imagem positiva que pode refletir em uma predisposição a considerar esta tipologia de projeto como de baixo impacto.

Contudo, conforme se verifica na literatura, os empreendimentos eólicos não são completamente favoráveis ao meio ambiente (Thygesen \& Agarwal, 2014) e chegam a causar impactos ambientais significativos (Baban \& Parry, 2001; Drewitt \& Lan- gston, 2006; Saidur et al., 2011; Tabassum-Abassi et al., 2014; Dai et al., 2015).

Vale destacar o expressivo crescimento deste setor no Brasil ao longo dos últimos anos. Desde 2012, o país encontra-se entre os 10 países com maior crescimento anual da capacidade instalada: em 2016 foi registrado a instalação de aproximadamente 2,0 GW (Global Wind Energy Council, 2017). Em fevereiro de 2018, a geração eólica brasileira atingiu a marca de $13 \mathrm{GW}$ de capacidade instalada, com 518 parques eólicos e mais de 6.600 aerogeradores em operação (Associação Brasileira de Energia Eólica, 2018).

\section{Metodologia}

O presente artigo, de natureza qualitativa, foi desenvolvido com base em pesquisa bibliográfica e documental, conforme preconizado por Gil (2002). A identificação do estado-da-arte do conhecimento científico sobre a ocorrência, prevenção e mitigação dos potenciais impactos ambientais de projetos de geração de energia eólica foi realizada a partir de revisão da literatura, empregando-se as bases de dados Scopus e Web of Science para localização de artigos relevantes.

O conjunto de impactos identificados constituiu a base para avaliação do conteúdo de 31 EIAs, elaborados entre 2010 e 2017 para empreendimentos eólicos no Ceará, estado pioneiro na geração de energia eólica no Brasil e ainda com grande destaque no setor.

A avaliação mencionada foi amparada pelo conceito de potencial de impacto, definido como o resultado da combinação entre a solicitação realizada pelo projeto e seus processos tecnológicos e a vulnerabilidade do meio (Sánchez, 2008). Sendo assim, os impactos identificados na literatura foram cotejados 
diante de informações referentes aos aspectos ambientais e sociais que compõem o território do estado e integrados a uma lista de verificação para avaliação do conteúdo dos EIAs.

A lista de verificação elaborada inclui 32 questões derivadas do levantamento dos impactos reportados pela literatura. As respostas "SIM" e "NÃO" refletem um aspecto positivo e negativo, respectivamente, verificado a partir do conteúdo dos EIAs.

A partir da caracterização de um conjunto de componentes socioambientais do estado do Ceará, realizada no item 4 deste artigo, todas as questões da lista de verificação foram consideradas aplicáveis ao contexto objeto de estudo.

Segundo informações fornecidas pelo órgão ambiental estadual do Ceará, Superintendência Estadual do Meio Ambiente (SEMACE), ao todo foram protocolados 74 EIAs de projetos de geração de energia, no âmbito do licenciamento ambiental estadual. Neste trabalho foram avaliados 31 EIAs, elaborados entre 2010 e 2017, os quais representam o total de EIAs completos disponíveis em formato digital na biblioteca da SEMACE, em julho de 2017.

\section{Impactos potenciais}

Nesta seção serão descritos os potenciais impactos socioambientais de projetos de geração de energia eólica, identificados a partir de revisão da literatura.

\subsection{Redução da diversidade e população de aves}

Foram registradas altas taxas de mortalidade de aves em várias partes do mundo (Orloff \&
Flannery, 1992; Hunt, 2002; Bevanger et al. 2010; González \& Ena, 2011; Ferrer et al., 2012; Dahl et al., 2015), principalmente associadas a projetos instalados em ou próximo de áreas regularmente usadas por grande número de aves para alimentação e repouso, e áreas de rotas migratórias (Travassos et al., 2005; Arnold \& Zink, 2011; Ouammi et al., 2012; Tabassum-Abassi et al., 2014).

Os registros de colisão de aves em aeroturbinas reportados na literatura são bastante variáveis (Drewitt \& Langston, 2006) e estão diretamente associados às espécies, à localização do empreendimento e a aspectos técnicos do projeto (Orloff \& Flannery, 1992; Barrios \& Rodríguez, 2004; Garthe \& Hüppop, 2004; Kuvlesky et al., 2007; Noguera et al., 2010; Braunisch et al., 2015). Portanto, são necessários estudos em escala local para previsão dos potenciais impactos (Tabassum-Abassi et al., 2014).

A ocorrência de impactos significativos sobre a avifauna não são restritos apenas à mortalidade por colisões com as estruturas dos aerogeradores, mas também à redução do êxito reprodutivo, alteração de comportamento de alimentação e ao afugentamento da fauna, em função de perturbação do habitat ou perda direta de habitat (Kaldellis et al., 2003; Dahl et al., 2012; Dai et al., 2015).

\subsection{Redução da diversidade e população de morcegos}

A redução da diversidade e populações de espécies de morcegos está relacionada ao aumento da taxa de mortalidade de espécimes, causada por colisão com as pás de aerogeradores ou barotrauma pela diferença de pressão do ar gerada pela rotação das pás (Kunz et al., 2007). 
A morte de morcegos em parques eólicos foi registrada em diversos países (Arnett et al., 2008; Baerwald \& Barclay, 2009; Voigt et al., 2012; Hayes, 2013). No entanto, a taxa de mortalidade varia espacialmente, dependendo das características ambientais (topografia, cobertura vegetal e uso do solo); das espécies, em função do comportamento de alimentação, altura de voo e hábitos migratórios; e das características dos aerogeradores, como altura das torres e layout do parque (Kunz et al., 2007; Arnett et al., 2008; Baerwald \& Barclay, 2009; Wang \& Wang, 2015).

As causas exatas para a mortalidade de morcegos em parques eólicos são complexas e ainda não são consenso na literatura (Rydell et al., 2010). Dentre as hipóteses, pode-se mencionar a implantação de parques eólicos em rotas migratórias (Arnett et al., 2008); atração pela emissão de ultrassom das turbinas (Arnett et al., 2005); atração de espécies que se alimentam de insetos que, por sua vez, se concentram nas proximidades de aerogeradores (Rydell et al., 2010); e o uso das torres como áreas de descanso ou pouso (Dai et al., 2015).

Em alguns países, para instalação de parques eólicos, foram definidas distâncias mínimas de habitats e locais para conservação de morcegos e exigida a avaliação de impacto ambiental (Kepel et al., $2011^{1}$ apud Kelm et al., 2014; Prefet de Lorrai$\mathrm{ne}^{2}, 2012$ apud Kelm et al., 2014).

\subsection{Impactos sobre a fauna terrestre: fragmentação/perda de habitats, redução da}

conectividade entre populações e redução da diversidade e população da fauna terrestre

A perda de habitats e redução da conectividade entre populações está associada tanto à alteração direta do uso do solo e redução da cobertura vegetal, como indiretamente com o afugentamento da fauna, alteração de rotas e de comportamento de algumas espécies (Braunisch et al., 2015; Dai et al., 2015).

Este impacto não está restrito apenas a aves e morcegos, englobando também animais terrestres (ou não voadores). Os impactos sobre a fauna terrestre causado por centrais eólicas ainda é pouco discutido na literatura, porém alguns estudos apontam o direto aumento da mortalidade, devido à destruição, alteração e fragmentação de habitats (Rabin et al., 2006; Santos et al., 2010; Helldin et al., 2012; Lovich \& Ennen, 2013; Lopucki \& Mróz, 2016), assim como redução da diversidade devido à fragmentação da vegetação, obstáculo à passagem e afugentamento da fauna (Lovich \& Ennen, 2013).

A emissão de ruído e vibração e o movimento das pás podem gerar distúrbios no ambiente e possivelmente tornar as áreas de entorno menos aptas como habitats (Helldin et al., 2012), refletindo em redução da abundância e diversidade de fauna (Lopucki \& Mróz, 2016). A abertura de vias de acesso aos aerogeradores pode facilitar o aumento de fluxo na região, causando o aumento da pressão nos habitats remanescentes (Lopucki \& Mróz, 2016) e mortalidade de animais terrestres por atropelamento (Lovich \& Ennen, 2013).

\footnotetext{
${ }^{1}$ KEPEL, A., M. CIECHANOWSKI, and R. JAROS. 2011. Wytyczne dotyczące oceny oddziaływania elektrowni wiatrowych na nietoperze [Guidelines for impact assessments for bats con- cerning wind farms]. Generalna Dyrekcja Ochrony Środowiska (GDOŚ), Warszawa

${ }^{2}$ PREFET DE LORRAINE. 2012. Schéma régional climat air énergie de Lorraine. Available at http://www.srcae.lorraine.gouv.fr/
} 
Tendo em vista a necessidade de extensas áreas, quando comparada a fontes de energia não renováveis (Brook \& Bradshaw, 2015), é possível que ocorram conflitos entre o crescimento da geração de energia e a conservação de habitats (Santangeli et al., 2016).

O processo de fragmentação de habitats em fragmentos menores ou isolados é uma das maiores preocupações para a conservação da biodiversidade e uma das principais causas de extinção de espécies (Fischer \& Lindenmayer, 2007). Neste caso, o enriquecimento de habitats remanescentes é apontado como importante medida compensatória (Braunisch et al., 2015; Dai et al., 2015).

A necessidade de extensas áreas pode ocasionar também o aumento da pressão sobre áreas protegidas e respectivo entorno e, consequentemente, impactos sobre a fauna (Ouammi et al., 2012). Na Holanda, por exemplo, foi proposta uma distância mínima entre parque eólicos e reservas naturais de 300 metros (Braunisch et al., 2015).

\subsection{Incômodo e danos à saúde da população devido ao aumento dos níveis de ruído}

O ruído proveniente das turbinas eólicas durante a operação tem duas origens: mecânica e aerodinâmica (Clarke, 1991; Lovich \& Ennen, 2013).

Os avanços tecnológicos permitiram uma redução significativa da geração de ruído mecânico (Wang \& Wang, 2015) e aerodinâmico (Jaskelevičius \& Užpelkiene, 2008). O ruído mecânico pode ser reduzido com o aprimoramento dos componentes de projeto, uso de isolamento adequado para as turbinas (Dai et al., 2015) e manutenção dos componentes (Davis \& Davis, 2007).
O ruído aerodinâmico pode ser subdividido em ruído de baixa frequência, ruído gerado por turbulência vertical e ruído gerado pela aerodinâmica da superfície das pás (Jaskelevičius \& Užpelkiene, 2008). A redução da espessura das pás e alteração da posição das estruturas dos aerogeradores em relação à direção do vento (upwind) também permitiram a redução do ruído aerodinâmico (Jaskelevičius \& Užpelkiene, 2008).

Apesar das lacunas de conhecimento em relação aos potenciais impactos na saúde da população vizinha (Leung \& Yang, 2012), alguns estudos apontam que o ruído aerodinâmico pode causar distúrbios no sono e perda da capacidade auditiva (Punch et al., 2010; Bakker et al., 2012) e que a baixa frequência pode causar incômodos, sintomas de estresse e dores de cabeça (Pedersen, 2011).

É importante destacar que os níveis sonoros podem ser relativamente baixos, porém a natureza pulsante causa maiores distúrbios em comparação com o mesmo nível de ruído constante (Pedersen \& Waye, 2006). Sendo assim, é possível que os limites legais de conforto acústico não sejam adequados para o ruído gerado por parques eólicos. Isto é, os níveis de ruído gerados podem ser facilmente medidos e previstos, porém o incômodo causado depende da percepção da população e de fatores subjetivos (Zamot et al., 2005).

O ruído ambiente e tipo de ocupação do entorno também podem influenciar na percepção do impacto (Tabassum-Abassi et al., 2014). Regiões com ocupação residencial são mais sensíveis ao aumento dos níveis de ruído (Tabassum-Abassi et al., 2014), enquanto a percepção desta alteração é menor em áreas com ruído ambiente elevado (Dai et al., 2015). 
Para estimar adequadamente o ruído resultante e prever o potencial impacto, é importante avaliar a direção do vento e posição das turbinas em relação ao receptor (Jaskelevičius \& Užpelkiene, 2008), o design das turbinas e a distância do receptor e a cumulatividade dos impactos (Markevičius et al., 2007; Ouammi et al., 2012; Wang \& Wang, 2015).

Alguns países estabeleceram distância mínima entre parques eólicos e habitações ou seguem recomendações de instituições de saúde, que variam de 300 a 1500 metros, ou foram definidas com base no diâmetro do rotor e altura da torre (Dai et al., 2015).

\subsection{Impactos visuais e alteração da paisa- gem}

A instalação de parques eólicos é descrita tanto como um impacto negativo na paisagem (Lothian, 2008; Langbroek \& Vanclay, 2012) como positivo em alguns estudos de percepção da população (Fokaides et al., 2014).

A literatura indica a percepção negativa em relação à instalação de aerogeradores e linhas de transmissão próximas de pontos paisagísticos, vistas panorâmicas e áreas com potencial arqueológico (Katsaprakakis, 2012), e associada a danos a atividades econômicas, como turismo e setor imobiliário (Mirasgedis et al., 2014).

Diversos fatores podem influenciar na magnitude e significância dos impactos como: características cênicas do local, topografia, paisagem entre as turbinas e os observadores (Katsaprakakis, 2012); número e tamanho das turbinas (Dimitropoulos \& Kontoleon, 2009; Tsoutsos et al., 2009); distância de áreas residenciais (Mirasgedis et al., 2014); material e cor das turbinas em contraste com o ambiente de fundo (Bishop \& Miller, 2007; Mirasgedis et al.,
2014); arranjo e espaçamento das turbinas (Mirasgedis et al., 2014).

Alguns estudos apontam que os parques eólicos tendem a ser vistos como impacto negativo de menor intensidade por visitantes ocasionais do que residentes locais (Molina-Ruiz \&Serrano, 2006), quando as pás estão em movimento (Thayer \& Freeman, 1987; Bishop \& Miller, 2007), quando as turbinas são do mesmo tamanho e dispostas com mesmo espaçamento (Thayer \& Freeman, 1987; Kaldellis et al., 2003).

Outra tipologia de impacto visual refere-se aos efeitos do reflexo ou sombreamento (shadow flicker) periódico da rotação das pás (Kaldellis et al., 2003), associado, principalmente, à proximidade com residências ou áreas de trabalho, em períodos de iluminação direta do sol (Kaldellis et al., 2003). $\mathrm{Na}$ Europa é recomendado um distanciamento de residências de 6 a 8 vezes o diâmetro do rotor (Kaldellis et al., 2003).

A avaliação dos impactos visuais é geralmente difícil, pois é subjetivo e depende da opinião e percepção dos receptores (Ouammi et al., 2012), da tipologia das turbinas, características ambientais locais e nível de exposição (Mirasgedis et al., 2014). Existem algumas abordagens para previsão e avaliação dos impactos, como a definição de zonas de visibilidade (Ladenburg \& Lutzeyer, 2012); estimativa de índice de design apropriado com base em fatores/parâmetros específicos (população nas áreas de entorno, número de turbinas) (Tsoutsos et al., 2009); previsão das futuras alterações da paisagem por meio de modelagem e fotomontagem (Hurtado et al., 2004). 


\subsection{Danos à população, meio ambiente e bens materiais devido à ocorrência de aci- dentes}

Os principais riscos de acidentes com aerogeradores estão relacionados ao desprendimento das pás, falhas elétricas, transporte e atividades construtivas, e incêndio (Clarke, 1991; Carneiro et al., 2013; Lovich \& Ennen, 2013; Caithness Windfarm Information Forum, 2018).

Segundo dados obtidos junto à plataforma Caithness Windfarm Information Forum (CWIF) para o período entre 2000 e junho de 2018 , foram registrados 2156 acidentes ou incidentes relacionados ao setor eólico, envolvendo falhas estruturais (principalmente nas pás), incêndio, atividades de transporte e lançamento de gelo, com 113 ocorrências fatais. Conforme estima a CWIF, o número de ocorrências registradas possivelmente corresponde a cerca de $10 \%$ do total de acidentes ocorridos de fato ${ }^{4}$. A gestão e análise dos riscos inerentes ao projeto é uma ferramenta importante para prevenção dos impactos e efeitos decorrentes destes acidentes (Carneiro et al., 2013).

\subsection{Perda de patrimônio histórico, cultural e arqueológico}

A instalação de projetos de geração de energia eólica de grande escala pode afetar sítios arqueológicos, atividades humanas e costumes tradicionais (Clarke, 2009; Delicado et al., 2016; Katsaprakakis \& Christakis, 2016). O risco de impacto pode ser alto em áreas cujo potencial histórico/arqueológico é desconhecido e localizado em áreas isoladas ou sem desenvolvimento de cultivo (Clarke, 2009).

Para prevenção de danos ao patrimônio histórico, cultural e arqueológico é importante a prospecção cuidadosa, antes do início da construção, o que possibilita a descoberta de sítios ou artefatos e eventuais modificações do projeto (Clarke, 2009).

\subsection{Alteração do uso e ocupação do solo e das atividades econômicas}

A instalação de grandes centrais de geração de energia eólica pode afetar as atividades existentes e alterar costumes tradicionais (Katsaprakakis \& Christakis, 2016).

Geralmente, a área efetivamente ocupada não é maior que $5 \%$ da área total do parque, o que permite o uso compartilhado com atividades agrícolas (Kaldellis et al., 2003). Contudo, o intenso crescimento do setor eólico pode reduzir a disponibilidade de grandes áreas para instalação de novos projetos e causar conflitos com o uso e ocupação do solo já existente onde há grande potencial eólico (Tabassum-Abassi et al., 2014).

As alterações na paisagem e danos ao patrimônio natural e arqueológico potencialmente causados pela instalação de parques eólicos pode indiretamente prejudicar o potencial turístico dessas áreas (Clarke, 2009; Devine-Wright \& Howes, 2010). Foram reportados impactos negativos ao turismo no Reino Unido (Devine-Wright \& Howes, 2010) e Grécia (Katsaprakakis \& Christakis, 2016), em

\footnotetext{
${ }^{3} \mathrm{http}: / / \mathrm{www}$. caithnesswindfarms.co.uk/AccidentStatistics.htm

${ }^{4} \mathrm{http}: / /$ www.caithnesswindfarms.co.uk/AccidentStatistics.htm
} 
regiões conhecidas pela beleza cênica e economia baseada no turismo.

Por outro lado, os efeitos sobre o turismo associados à construção de parques eólicos não são reportados apenas como negativos na literatura, tendo sido identificados também como promotores do desenvolvimento de nova forma de turismo (Frantál \& Kunc, 2011; Frantál \& Urbánková, 2017), denominada por Frantál \& Urbánková (2017) como "turismo de energia".

A instalação de parques eólicos pode acarretar também flutuação do valor imobiliário de propriedades próximas, tendo sido registrados tanto valorização quanto desvalorização (Petrova, 2013), bem como conflitos fundiários (Brannstrom et al., 2018).

A instalação de parques eólicos pode implicar em limitação de acesso a extensas áreas e em impactos sobre comunidades tradicionais e atividades econômicas, devido ao bloqueio de trilhas e caminhos de acesso a áreas tradicionalmente usadas como fonte de renda ou para recreação (Brown, 2011; Loureiro et al., 2015; Brannstrom et al., 2017).

\subsection{Outros impactos associados às ativida- des construtivas}

Alguns impactos reportados na literatura estão associados às atividades construtivas de um modo geral, e devem ser considerados no âmbito da AIA, pois podem ser significativos. A construção de parques eólicos envolve atividades que podem intensificar processos erosivos e, eventualmente, assoreamento dos corpos d'água, e alteração da qualidade dos recursos hídricos e solo, pela desti- nação ou manejo inadequado de efluentes, resíduos e óleo (Dai et al., 2015).

Durante a construção, também podem ser causadas interferências e alterações no ecossistema terrestre e sistema hidrológico, cuja significância dos impactos dependerá da vulnerabilidade do ecossistema, técnicas construtivas e medidas mitigadoras (Kaldellis et al., 2003). Como medidas de controle e redução dos impactos pode-se citar a execução de sistemas de drenagem (Kaldellis et al., 2003; Lovich \& Ennen, 2013) sobretudo nas vias de acesso e recuperação da cobertura vegetal (Gong, $2004^{5}$ apud Dai et al., 2015).

Durante as atividades construtivas há intensa movimentação de veículos, equipamentos e mão de obra que podem causar incômodos à população do entorno da área de obras e vias de acesso, bem como danos ao patrimônio material (residências, vias de acesso e demais edificações) e acidentes (Brown, 2011).

\subsection{Interferência eletromagnética em meios de comunicação e sistemas de navegação}

O campo eletromagnético de uma turbina eólica pode causar interferências eletromagnéticas nos meios de comunicação e navegação (Clarke, 1991; Zamot et al, 2005; Dai et al., 2015). Tais efeitos foram minimizados com o uso de materiais sintéticos para fabricação das pás (Steele, 1991), tendo sido registrados principalmente para a primeira geração de turbinas eólicas com pás metálicas (Tabassum-Abassi et al., 2014).

\footnotetext{
${ }^{5}$ Gong J. The construction of wind turbine generator system. In: Gong J, editor. A technical guideline for wind turbine generator system. Beijing, China: China Machine Press; 2004. p. 120-4
} 
Apesar do campo eletromagnético de uma turbina eólica ser fraco e restrito à área direta de entorno (Katsaprakakis, 2012), a identificação de fontes de transmissão de dados de comunicação e navegação que possam ser afetadas é importante na fase de planejamento e para prevenção de potenciais impactos. Em alguns países, foram propostas distâncias mínimas a serem observadas entre parques eólicos e estações de meios de telecomunicação (Tabassum-Abassi et al., 2014).

\subsection{Alterações no microclima}

A operação de turbinas eólicas pode alterar o clima local e regional (Roy \& Traiteur, 2010; Roy, 2011; Walsh-Thomas et al., 2012; Zhou et al., 2012) e influenciar de alguma forma a produção agrícola (Tabassum-Abassi et al., 2014) ou clima global (Keith et al., 2004).

Zhou et al. (2012) identificaram a tendência de aumento da temperatura em $0,72^{\circ} \mathrm{C}$ por década, sendo a variação mais intensa no período noturno. Roy \& Traiteur (2010) também identificaram a tendência de aumento da temperatura no período noturno e esfriamento durante o dia, devido à turbulência criada pela rotação das pás e o efeito de mistura vertical do ar próximo à superfície do solo.

Roy (2011) aponta possíveis efeitos na hidrometeorologia, isto é, efeitos na temperatura e umidade do ar e fluxos de calor latente, que podem se estender por até $23 \mathrm{~km}$ a favor do vento. Keith et al. (2004), sugerem que, no limite, o desenvolvimento da energia eólica em larga escala poderia afetar o clima em escala global, pela redução de energia cinética e alteração do transporte turbulento na camada limite da atmosfera.
A literatura aponta a necessidade de estudos mais aprofundados para que sejam avaliados de modo conclusivo os potenciais efeitos e impactos sobre as condições climáticas e hidrometeorológicas (Roy, 2011). Porém, as evidências disponibilizadas até o momento indicam a necessidade de levantamento de dados prévios e monitoramento dos impactos efetivos de parques eólicos já em operação.

\subsection{Impactos durante o descomissionamen- to das instalações ou repotencialização}

A vida útil de parques eólicos é, em média, de 20 anos, após o qual existem basicamente duas possibilidades: repotencialização ou descomissionamento (Ortegon et al., 2013). Ambas envolvem o transporte dos componentes e perturbações no ambiente semelhantes às da etapa de construção, com desmonte, separação, recuperação e manejo ou destinação dos componentes do sistema de geração eólica (Ortegon et al., 2013).

Cerca de onze mil turbinas eólicas nos Estados Unidos alcançaram o fim da vida útil até 2013 e estima-se que até 2030 seriam cerca de vinte e nove mil (Ortegon et al., 2013), o que ilustra a relevância do tema para a prevenção de impactos ambientais associados.

A repotencialização é uma alternativa para manter a atividade em operação e possibilita aumento da geração de energia em menor área, por meio da substituição das turbinas antigas por turbinas mais modernas e mais potentes (Goyal, 2010).

Para redução do volume de resíduos gerados e recuperação de material, esta etapa pode incluir a reciclagem, recondicionamento, reuso de alguns componentes e remanufatura completa do sistema eólico (Ortegon et al., 2013). Esta estratégia é comumente aplicada na Europa, especialmente na Alemanha e Dinamarca (Goyal, 2010). 


\subsection{Impactos cumulativos}

A combinação dos efeitos de múltiplas ações ou empreendimentos pode criar maiores pressões sobre o meio ambiente do que a simples soma individual das partes, em um processo associado à incidência de impactos sobre um mesmo componente ambiental (Canter \& Kamath, 1995). Assim, o intenso aumento do número de empreendimentos de geração de energia eólica observado nos últimos anos suscita preocupações com relação à cumulatividade dos impactos.

Os efeitos cumulativos de empreendimentos eólicos apontados na literatura estão especialmente relacionados a impactos negativos sobre a avifauna e quiropterofauna (Stewart et al., 2007; Masden et al., 2010), alteração de habitat (Fox et al., 2006), geração de incômodo pelo aumento dos níveis de ruído e alteração da paisagem (Dimitropoulos \& Kontoleon, 2009; Tsoutsos et al., 2009; Josimovic \& Pucar, 2010). Wilson et al. (2010) são enfáticos ao relacionar a ocorrência de impactos cumulativos ao planejamento deficiente da localização e layout de centrais eólicas em relação às áreas de entorno.

\section{Aspectos ambientais relevantes para a avaliação de impactos de empreendimentos eólicos no estado do Ceará}

O conceito de potencial de impacto está associado ao resultado da "combinação entre a solicitação (característica inerente ao projeto e seus processos tecnológicos) e a vulnerabilidade do meio" (Sánchez, 2008, p. 113). Sendo assim, entende-se que a revisão da literatura descrita na seção anterior do presente artigo permitiu identificar as pressões (solicitações) exercidas pelos empreendimentos de geração de energia eólica e os principais componentes ambientais potencialmente afetados.

No caso do estado do Ceará, a identificação da distribuição espacial dos componentes ambientais potencialmente afetados por empreendimentos eólicos permite compreender a vulnerabilidade do meio aos impactos potenciais, especialmente relacionados à biodiversidade - representada pela avifauna, quiropterofauna e espécies ameaçadas de extinção, assim como pelas áreas protegidas e demais áreas de interesse para conservação - comunidades locais, povos indígenas e populações tradicionais.

A Figura 1 apresenta a localização dos parques eólicos e áreas sensíveis no estado do Ceará, ilustradas pelas Áreas Prioritárias para Conservação da Biodiversidade, UCs, Terras Indígenas, Comunidades Quilombolas e áreas com a presença de aves migratórias.

\subsection{Aves, morcegos e espécies ameaçadas}

É importante destacar que a perda de biodiversidade constitui um dos aspectos mais críticos para o sistema ambiental planetário e que, portanto, qualquer agravamento neste quadro induzido por atividades humanas deve ser evitado (Rockström et al., 2009).

O Brasil é considerado um dos países de megadiversidade biológica, com mais de 4400 espécies de fauna terrestre (mamíferos, aves, répteis e anfíbios), das quais cerca de $10 \%$ são consideradas ameaçadas, segundo dados da União Internacional para Conservação da Natureza (UICN) (Instituto Chico Mendes de Conservação da Biodiversidade - ICMBIO, 2016a). No caso do estado do Ceará, 
as informações coletadas indicam o registro de 27 espécies de fauna terrestre ameaçadas/vulneráveis à extinção, sendo 6 espécies de mamíferos, 15 espécies de aves, 4 espécies de répteis e 2 de anfíbios (Instituto de Pesquisa e Estratégia Econômica do Ceará, 2016).

Em relação à diversidade de aves, o Brasil é o segundo país do mundo em número de espécies identificadas, com 1919 espécies (Piacentini et al., 2015). São reconhecidas como migratórias ao menos 197 espécies e existem cinco rotas principais de migração; duas cruzam a região nordeste, a "Rota Atlântica" e a "Rota Nordeste" (ICMBIO, 2016a).

No Ceará, os registros existentes indicam uma significativa riqueza de aves, com mais de 350 espécies oficialmente registradas (Fernandes-Ferreira et

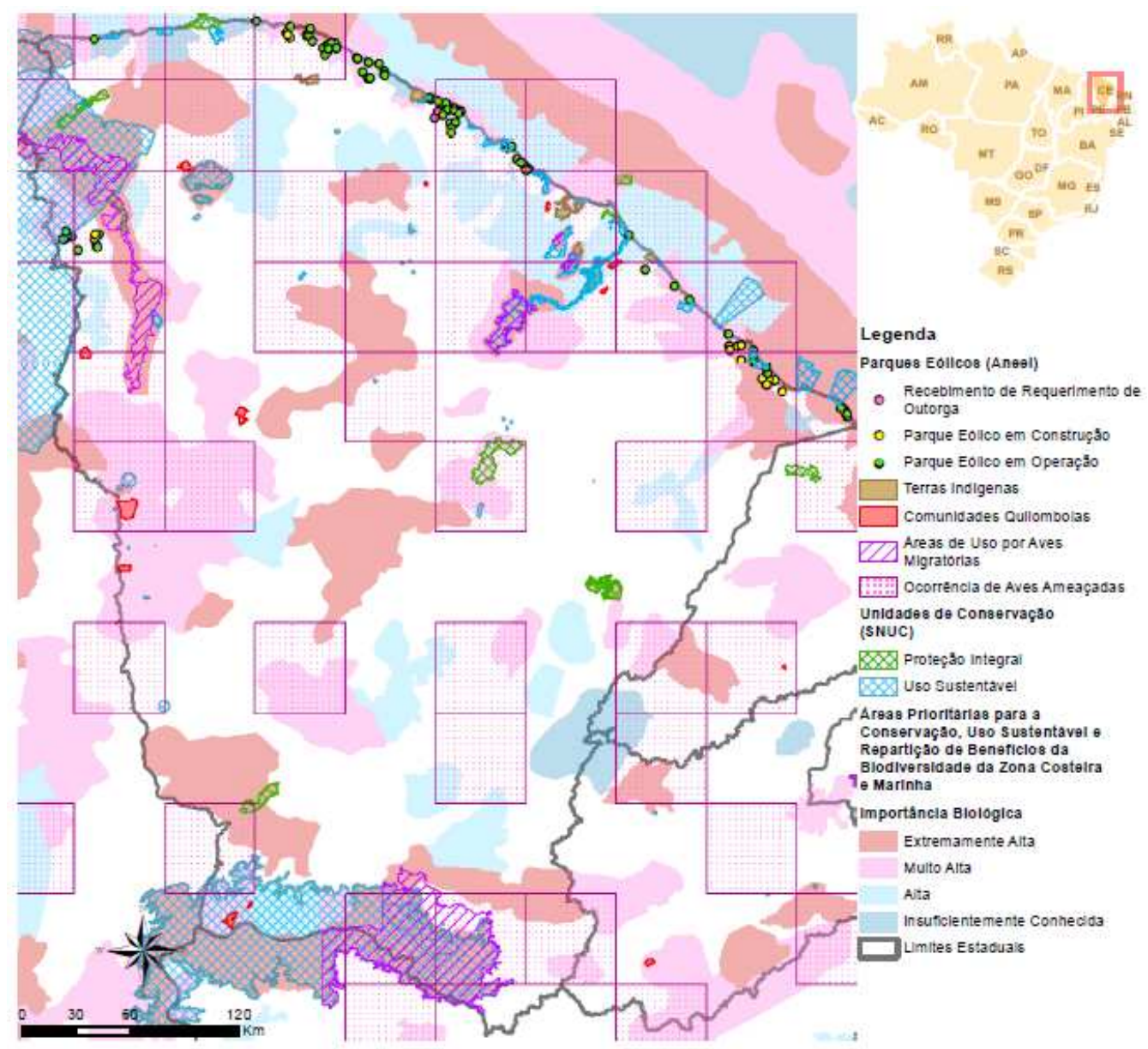

FIGURA 1 - Localização dos parques eólicos e áreas de sensibilidade ambiental.

FONTE: elaborado por Ricardo Fogarolli com dados georreferenciados disponíveis online (Birdlife International, 2018; FUNAI 2018; INCRA, 2018; Brasil, 2018a). 
al., 2012), 6 áreas de reprodução de aves migratórias e 3 áreas com concentração de espécies migratórias (ICMBIO, 2016b). Deve-se destacar, no entanto, que o conhecimento científico sobre as populações de aves migratórias no Brasil apresenta grandes lacunas (Somenzari et al., 2018), assim como sobre a avifauna no estado do Ceará (Girão et al., 2008).

Em relação à quiropterofauna, o Brasil abriga cerca de $15 \%$ das espécies conhecidas no mundo e é o segundo país com maior riqueza (Bernard $e t$ al., 2011), com 174 espécies, das quais 77 foram registradas na Caatinga, bioma presente em todo o estado do Ceará (Paglia et al., 2012).

Apesar do conhecimento sobre a ocorrência e distribuição das espécies de morcegos no Brasil ter aumentado nas últimas décadas, a carência de informações ainda é significativa. À época em que apresentaram seus resultados, Bernard et al. (2011) apontavam que cerca de $60 \%$ do território não apresentava nenhum dado a esse respeito, incluindo-se apenas $10 \%$ do território do bioma da Caatinga como possuidor de alguma informação sobre a presença e distribuição de espécies de morcegos. Complementarmente, informações sobre impactos de projetos de geração de energia eólica sobre morcegos no Brasil ainda são escassas e insuficientemente avaliadas nos processos de licenciamento ambiental (Bernard et al., 2014).

\subsection{Vegetação nativa, áreas protegidas e demais áreas de interesse para conservação}

No Brasil as áreas protegidas englobam as Área de Preservação Permanente (APP) e Reserva Legal, regulamentadas pelo Código Florestal (Lei 12.651/2012), e Unidades de Conservação (UC), conforme a Lei no 9.985/2000. O estado do Ceará possui 12 UCs federais, 26 estaduais, 4 municipais e 34 Reservas Particulares do Patrimônio Natural (Brasil, 2018b).

De modo particularmente importante para os projetos eólicos instalados no Ceará, destaca-se que a Resolução CONAMA no 303 de 2002 estabelece como APP as áreas situadas em dunas, locais de refúgio ou reprodução de aves migratórias, locais de refúgio ou reprodução de exemplares da fauna ameaçadas de extinção, e praias.

A construção de empreendimentos de geração de energia eólica em dunas (móveis ou fixas) tem causado interferência na dinâmica de movimentação dos campos de dunas, lagoas interdunares e água subterrânea, como resultado das atividades de terraplanagem, supressão da vegetação fixadora, fixação das dunas e demais atividades construtivas para implantação de vias de acesso (Lima, 2008; Meireles, 2011; Moura-Fé \& Pinheiro, 2013).

Os campos de dunas são paisagens ambientalmente frágeis, sensíveis a todo e qualquer tipo de intervenção (Moura-Fé \& Pinheiro, 2013) e alterações morfológicas nesse sistema provocam reflexos no nível hidrostático e alterações no leito sazonal das lagoas interdunares (Meireles, 2011). As interferências na dinâmica de campos de dunas em Camocim (CE) causaram o desaparecimento de uma lagoa utilizada como local de lazer e fonte de alimento da comunidade local (Brannstrom et al., 2017).

Segundo mapeamento realizado pelo Serviço Florestal Brasileiro, cerca de 57\% do território do estado do Ceará era coberto por florestas naturais, principalmente, por Savana Estépica - Caatinga ( $87,7 \%$ da cobertura florestal), e apenas $7 \%$ situado em alguma Unidade de Conservação (Brasil, 2016). 
Também configuram como áreas sensíveis e de potencial interesse para a fauna as Áreas Prioritárias para Conservação da Biodiversidade, regulamentadas pelo Decreto 5.092/2004. As áreas prioritárias para conservação não representam restrição legal para instalação de empreendimentos eólicos, porém devem ser objeto de estudo detalhado, uma vez que podem ter sido delimitadas visando à criação de novas Unidades de Conservação ou à conservação de espécies ameaçadas ou vulneráveis, dentre outros fins.

A importância da conservação dessas áreas é refletida nos números de áreas prioritárias (282) e de espécies alvo de conservação (350 plantas, 65 aves, 31 mamíferos, 30 répteis, 22 anfíbios, 126 peixes e 12 habitats costeiros), propostas na $2^{\mathrm{a}}$ atualização das Áreas e Ações Prioritárias para Conservação, Uso Sustentável e Repartição dos Benefícios da Biodiversidade do bioma da Caatinga, realizada em 2015 e aprovada pela Portaria N²23, de 21 de junho de 2016 (Brasil, 2015).

\subsection{Comunidades locais e tradicionais e patrimônio histórico, cultural e arqueológico}

O Decreto 8.750/2016 estabelece no Art. $4^{\circ}$ que o conjunto de comunidades ou populações tradicionais integra diferentes grupos: indígenas, quilombolas, caiçaras, pescadores tradicionais, ribeirinhos. No estado do Ceará existem 50 comunidades quilombolas certificadas pela Fundação Palmares até abril de 2018 (Fundação Cultural Palmares, 2018) e 14 comunidades indígenas, integrando uma população total próxima a $30 \mathrm{mil}$ pessoas (Instituto de Medicina Integral Professor Fernando Figueira, 2015).
Contudo, o processo de regularização das terras indígenas (TI) no estado é moroso (Lima \& Marquesan, 2017), e existem no Ceará apenas 2 TI regularizadas e a população indígena tem sofrido diversos problemas decorrentes da utilização ilegal de suas terras para exploração de recursos naturais, construção de residenciais e complexos turísticos (Lima \& Marquesan, 2017).

A proteção do patrimônio histórico e artístico nacional foi regulamentada pelo Decreto-Lei $n^{\circ}$ 25/1937 e pela Constituição Federal de 1988 (Art. 215 e 216), com foco no conjunto dos bens móveis, imóveis e culturais de natureza material e imaterial da população.

O Ceará possui quatro conjuntos urbanos tombados (cidades históricas) e um rico patrimônio que inclui 528 sítios arqueológicos cadastrados até dezembro de 2014 (Instituto do Patrimônio Histórico e Artístico Nacional - IPHAN, 2018). Há ainda no estado cerca de 60 processos de tombamento em andamento (IPHAN, 2017), o que indica o constante descobrimento de novos bens. $\mathrm{O}$ alto potencial arqueológico na região e a ocorrência de impactos negativos significativos sobre este componente foi constatado durante o processo de licenciamento de dois complexos eólicos nos municípios de Taíba (CE) e Aracati (CE) (Lima, 2008).

Para prevenção dos impactos às comunidades locais, muitos países definiram distância mínima entre parques eólicos e residências (Dai et al., 2015). Porém, no Brasil não há regulamentação específica sobre o tema. O único diploma legal que estabelece alguma condição de distância para parques eólicos é a Resolução Conama 462/2014, que determina a necessidade de previsão dos índices de ruído nos estudos ambientais prévios para empreendimentos a menos de 400 metros de residências isoladas ou 
comunidades. A mesma resolução define que os índices de ruído devem atender às normas da $\mathrm{ABNT}$ na área de influência direta do empreendimento.

No Ceará, Brannstrom et al. (2017) identificaram desconforto dos moradores em função do ruído gerado pelas turbinas de um parque eólico instalado a apenas 200 metros de uma comunidade de 20 famílias, no município Camocim - CE.

Outro fator de incômodo e danos à saúde em áreas próximas de parques eólicos no Ceará é a emissão de material particulado (poeira), principalmente durante a etapa construtiva, devido ao trânsito de veículos e equipamentos pesados em vias não pavimentadas (Brown, 2011).

Em parques eólicos no Ceará também foram identificados problemas enfrentados pelas comunidades devido à privatização de acessos e supressão da vegetação, o que acarretou alterações no ecossistema local (mangue) e dificuldade de acesso a áreas de pesca e coleta de recursos naturais, exploradas para subsistência pela população local (Brown, 2011; Loureiro et al., 2015; Brannstrom et al., 2017).

Loureiro et al. (2015) conduziram um estudo de caso em Acaraú (CE), onde havia (em março de 2015) ao todo 199 turbinas eólicas, e previsão de instalação de outras 92 turbinas. De acordo com este estudo, a comunidade considera a instalação de parques eólicos em dunas um impacto negativo na paisagem, pois reduz o potencial paisagístico e turístico da região.

Em função de impactos na paisagem, stakeholders do setor de turismo têm pressionado o governo nos níveis federal e estaduais para limitar a instalação de parques eólicos em áreas com menor potencial de afetar o turismo (Brown, 2011). Por outro lado, Araújo (2014) identificou, por meio de questionário, alto índice de apoio de turistas e visitantes domésticos $(71,8 \%)$ ao desenvolvimento da energia eólica em dois famosos pontos turísticos do Ceará (praias de Canoa Quebrada e Jericoacoara).

\section{Verificação do conteúdo dos Estudos de Impacto Ambiental}

Os resultados da aplicação da lista de verificação para análise do conteúdo dos EIAs são apresentados na Tabela 1 .

De modo geral, verifica-se uma aproximação razoável com o estado-da-arte do conhecimento relacionado aos impactos potencialmente significativos derivados da implantação de parques eólicos: 17 dos 33 critérios estabelecidos verificados estavam presentes em pelo menos 20 (de 31) EIAs. Apesar disso, os resultados apontam lacunas de informações importantes sobre a vulnerabilidade dos componentes socioambientais potencialmente afetados por projetos de geração de energia eólica e de propostas de monitoramento dos potenciais impactos.

Os impactos sobre a avifauna foram escopo de todos os EIAs avaliados, porém foram observadas deficiências em relação às informações da linha de base. Um número considerável de EIAs não apresentou o levantamento de potenciais áreas de reprodução, pouso ou alimentação (23 EIAs, ou 74\% — questão i.2), assim como informações sobre rotas migratórias (11 EIAs, 35\% — questão i.3) e proposta de monitoramento de colisão de aves durante a operação (8 EIAs, $26 \%$ - questão i.4).

Cerca de 55\% dos EIAs (17 EIAs) não apresentaram informações primárias sobre espécies de morcegos (questão ii.1) e, assim como para avifauna, não foi previsto o monitoramento da taxa de mortalidade 
de morcegos (questão ii.2) em 8 EIAs (26\%). Tendo em vista a extensa literatura científica sobre impactos sobre aves e morcegos, a ausência de tais informações nos EIAs deve ser considerada uma omissão relevante.

Identificou-se que 22 projetos interferem em fragmentos de vegetação natural (questão iii.6), sendo que apenas 4 propõem medidas de enriquecimento de outros habitats (questão iii.7). Além disso, em 7 EIAs não foram apresentadas informações suficientes para verificar se haveria ou não interferências em fragmentos vegetais (questão iii.6). Este resultado também configura uma limitação relevante para a compreensão da vulnerabilidade do meio ambiente afetado pelos projetos analisados e, consequentemente, para a avaliação dos impactos associados.

Verificou-se que 13 projetos resultam em interferência em APP (questão iii.4), principalmente representadas por APP de corpos d'água, área de vegetação fixadora de dunas ou áreas de dunas; e 24 EIAs informaram a ocorrência de intervenções diretas em Áreas Prioritárias para Conservação da Biodiversidade (questão iii.5). $\mathrm{O}$ alto número de projetos aprovados nessas áreas indica que as Resoluções CONAMA 01/86 e 369/06 não estão sendo aplicadas satisfatoriamente, no que diz respeito à análise de alternativas tecnológicas e de localização de projeto e escolha da mais favorável, levando-se em conta a necessidade de demonstrar a inexistência de alternativas locacionais que evitem interferências sobre APPs.

A geração de ruído foi incluída no escopo de todos os EIAs avaliados, porém foram identificadas deficiências na qualidade das informações necessárias para avaliação da magnitude e significância dos potenciais impactos. 10 EIAs não apresentaram o levantamento dos níveis de ruído ambiente (questão iv.1); 20 EIAs não previram os níveis de ruído nas áreas de entorno durante a operação (iv.2); e 15 EIAs não informaram a distância entre o projeto e as residências mais próximas (iv.3). Por outro lado, apenas 2 EIAs não previram o monitoramento dos níveis de ruído durante a operação (iv.4), apesar de terem proposto o monitoramento durante a construção, considerando saúde e segurança ocupacional.

Segundo informações apresentadas nos EIAs, na maioria dos casos, os empreendimentos localizam-se em áreas rurais ou faixas de praias, com baixa densidade populacional e distante de centros urbanos ou de alta concentração populacional. Porém, mesmo nestes casos, observa-se a presença de residências isoladas ou pequenas comunidades rurais e, portanto, há vulnerabilidade aos impactos associados à geração de ruído na operação.

Apesar da importância do tema, apenas 5 EIAs elaboraram modelagem ou fotomontagem do cenário futuro da área com a instalação do projeto (questão v.1).

Nove EIAs avaliados não apresentaram caracterização de grupos e povos tradicionais (questão vii.3), o que também implica em uma deficiência significativa. Dentre os 22 EIAs avaliados que apresentaram levantamento de comunidades tradicionais, 3 interferem em áreas de populações tradicionais, sendo $2 \mathrm{em}$ comunidades quilombolas e $1 \mathrm{em}$ terra indígena. Nos 3 casos, não são estudadas outras alternativas locacionais para prevenção do impacto, tendo sido informado que será realizado um outro estudo detalhado, isto é, o EIA se omite em detalhar os potenciais impactos do projeto sobre tais comunidades, pois serão escopo de estudo posterior.

Cumpre esclarecer que para aplicação da questão vii.3 foram consideradas apenas as comunidades indígenas e quilombolas, para as quais foram regulamentados territórios tradicionais e existem requisitos mínimos legais para fins de licenciamento (Portaria 
TABELA 1 - Resultados da aplicação da lista de verificação para empreendimentos eólicos.

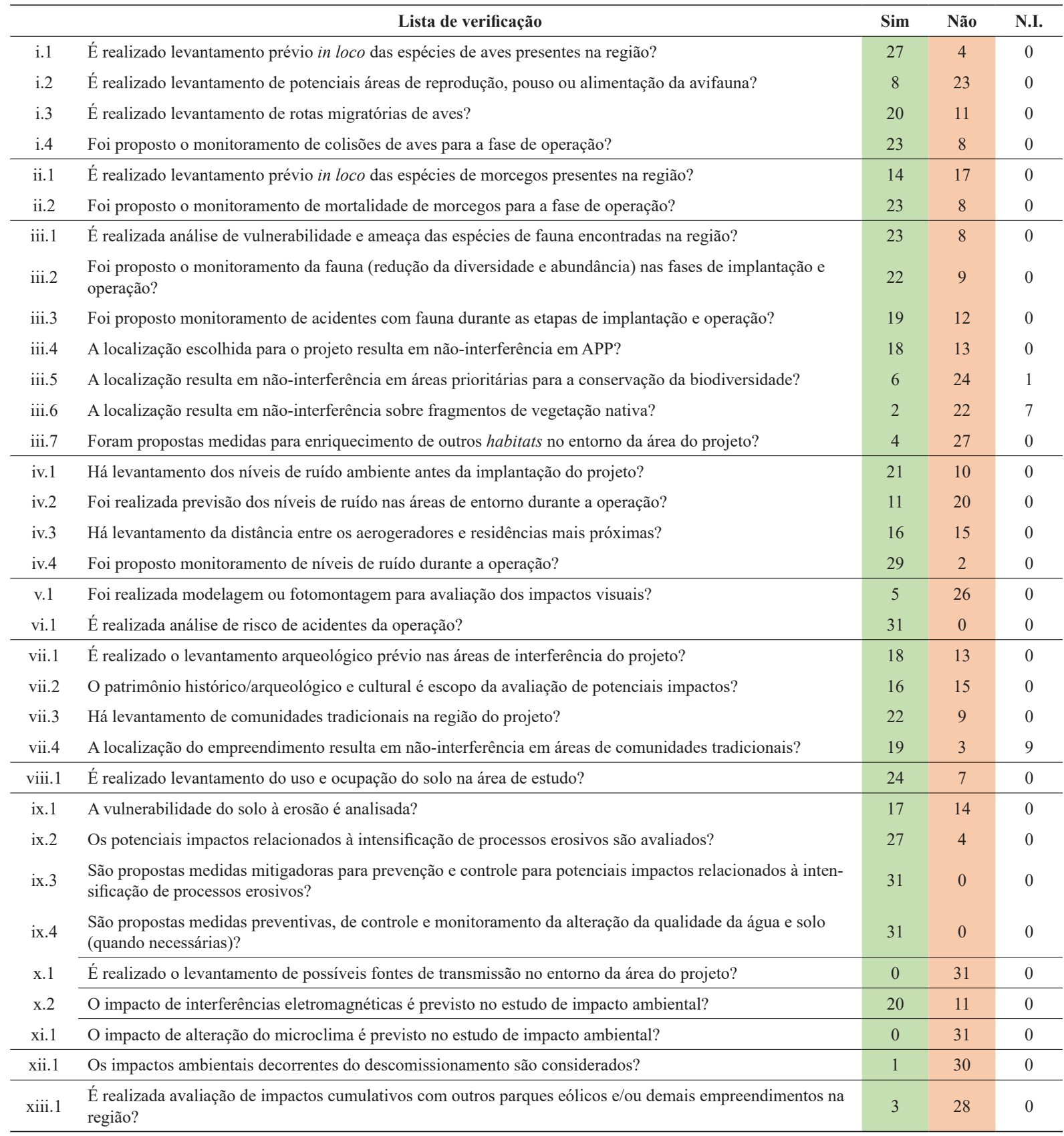

FONTE: elaborado pelos autores.

NOTA: N.I. - Não informado. 
Interministerial $n^{\circ}$ 60/2015). Porém, destaca-se que todos os grupos e povos tradicionais, importantes para o contexto do local de estudo, devem ser incluídos no âmbito da AIA, a exemplo das comunidades pesqueiras do Ceará (Lima, 2008).

Todos os EIAs apresentaram alguma análise de risco de acidentes durante a operação (questão vi.1) e medidas mitigadoras para os impactos relacionados à intensificação de processos erosivos e alteração da qualidade da água e do solo (questões ix.3 e ix.4). Este resultado indica um aspecto positivo no caso das eólicas pela associação a potenciais impactos de natureza similar decorrentes de outras tipologias de projeto.

Também foram observadas deficiências nas informações da linha base e avaliação dos impactos sobre a qualidade do solo e água (questões xi). Catorze EIAs não analisaram a vulnerabilidade do solo e 4 EIAs não contemplaram os impactos relacionados à intensificação de processos erosivos na avaliação, apesar de proporem programas de controle para os mesmos.

O resultado das questões viii.1 e xi.1 indicam deficiência na elaboração da linha de base para avaliar os impactos de interferências nas atividades econômicas devido à alteração do uso do solo e eventuais alterações no microclima. Sete EIAs não apresentaram levantamento do uso e ocupação da área de influência e nenhum EIA identificou o potencial impacto de alteração do microclima no escopo da avaliação. Apenas 18 EIAs (58\%) realizaram levantamento prévio do patrimônio histórico arqueológico.

A ausência de informações relevantes para avaliação dos impactos também foi verificada nas questões x. 1 e x.2, uma vez que 20 EIAs identificaram como potencial impacto a ocorrência de interferências eletromagnéticas em meios de comunicação, porém nenhum executou o levantamento de fontes de transmissão no entorno.

Estes resultados são um indicativo de baixa conexão entre as etapas de AIA e baixo nível de atendimento aos princípios da IAIA (1999) referentes à condução de um processo sistemático que considere todas as informações relevantes do meio afetado.

A questão xii.1, referente aos impactos decorrentes das atividades de desativação e descomissionamento dos parques eólicos, foi objeto de avaliação de apenas 1 EIA. No entanto, o programa de desativação apresentado neste EIA não contemplou medidas para destinação adequada dos componentes ou repotencialização do parque, conforme indicado por Ortegon et al. (2013). Seguindo os princípios de AIA, é desejável que a etapa de desativação dos parques seja escopo dos estudos ambientais, uma vez que o processo de AIA deve considerar todo ciclo de vida de atividades que possam causar impactos significativos (IAIA, 1999).

A avaliação de impactos cumulativos é considerada a área menos desenvolvida na AIA (Morgan, 2012). A cumulatividade dos impactos causados por projetos de geração de energia eólica é apontada para diversos componentes ambientais (Tsoutsos et al., 2009; Masden et al., 2010; Zhou et al., 2012). Contudo, apenas 3 EIAs realizaram alguma análise de cumulatividade. No estado do Ceará, observa-se intenso crescimento da capacidade instalada de fonte eólica, com concentração de parques eólicos em diversos municípios, evidenciando a importância da avaliação de impactos cumulativos. 


\section{Discussão}

Os resultados deste trabalho indicam que não há padronização do escopo dos EIAs elaborados para projetos de geração de energia eólica no estado do Ceará, tendo sido observadas tanto boas práticas como deficiências importantes nos estudos avaliados.

A ausência de informações importantes para prevenção e avaliação de impactos potenciais, considerando o contexto nacional e estadual, indica que a avaliação de impactos não foi realizada com base em informações adequadas e suficientes. Além disso, observou-se alguns casos de baixa conexão entre as etapas da AIA, com a identificação de impactos sem informações de linha base e proposição de medidas mitigadoras para impactos não identificados nos EIA. Desse modo, os impactos potenciais não têm sido considerados satisfatoriamente nos níveis de planejamento para prevenção (alternativas locacionais) ou para mitigação e compensação.

Gorayeb et al. (2018) identificaram completa ausência de informações no estudo de impacto ambiental de um parque eólico em Camocim - CE sobre uma comunidade tradicional localizada a 200 metros das instalações eólicas, entre outras deficiências. A omissão de tais informações limitou a compreensão dos tomadores de decisão em relação aos potenciais impactos sociais do projeto e, possivelmente, acelerou o processo de aprovação do licenciamento ambiental (Gorayeb et al., 2018).

A qualidade, o rigor e a clareza das informações apresentadas no âmbito do processo de AIA podem contribuir para um processo decisório robusto e para a efetividade do instrumento como um todo (Fischer, 2007; Chanchitpricha \& Bond, 2013). A determinação das questões importantes e que devem ser avaliadas no processo de AIA, conhecida como etapa de escopo (Borioni et al., 2017), é fundamental e pode influenciar a qualidade dos estudos ambientais (Mcgrath \& Bond, 1997; Baxter et al., 2001) e o fortalecimento do instrumento de um modo geral (Canter \& Ross, 2014).

No entanto, a elaboração de estudos com amplo escopo e sem foco nas questões chave tem sido alvo de crítica, considerados de pouca contribuição para a tomada de decisão e um limitante para a efetividade da AIA (Baxter et al., 2001; Canter \& Ross, 2014).

A descrição genérica sobre a área potencialmente afetada tem sido relacionada a uma descrição insuficiente dos potenciais impactos (Peterson, 2010). Borioni et al. (2017) verificaram que essa abordagem tem sido praticada no Brasil, conduzida sem a formalização de diretrizes ou guias.

Wynne (1992) destaca o papel das instituições envolvidas na implementação de políticas ambientais em relação à validação do conhecimento científico, a partir do exercício do que o autor denomina de 'controle do mundo social', tendo em vista o distanciamento intrínseco entre um (conhecimento científico) e outro ('mundo social') ocasionado - dentre outros fatores - pela incerteza inerentemente associada ao conhecimento de um dado processo ou fenômeno ambiental. Deste modo, é importante que os EIAs em que foram verificadas boas práticas sejam compreendidos pelo órgão ambiental como exemplos positivos para o aprimoramento do conteúdo dos EIAs futuros e, deste modo, promover uma aproximação entre conhecimento científico e a prática da AIA. 


\section{Conclusões}

O estado do Ceará apresenta componentes ambientais de alta sensibilidade aos efeitos e pressões decorrentes de projetos eólicos, sobretudo em relação à avifauna, quirópteros e populações e comunidades tradicionais. Desse modo, há possibilidade de ocorrência de impactos significativos, o que, portanto, destaca a importância da AIA para garantir a conservação ambiental e bem-estar das comunidades locais.

No entanto, de modo geral, foram evidenciadas lacunas significativas entre a prática de AIA e o conhecimento científico sobre os potenciais impactos de projetos de geração de energia eólica. Os resultados deste trabalho indicam o distanciamento entre a prática de AIAe a ciência. Nesse sentido, observa-se também que o processo de AIA objeto de estudo não está em consonância com princípios de boas práticas de AIA, em relação à aplicação das melhores metodologias e técnicas científicas praticáveis e o fornecimento de informação suficiente e confiável.

Os componentes socioambientais do estado do Ceará indicam vulnerabilidade em relação aos efeitos e pressões decorrentes de projetos eólicos e, portanto, há possibilidade de ocorrência de impactos significativos. Sendo assim, o presente estudo indica que, se foram consideradas apenas as informações apresentadas nos EIAs, as decisões estão sendo tomadas com base em informações insuficientes e inadequadas.

Por outro lado, a identificação de casos pontuais de boas práticas destaca a responsabilidade dos órgãos ambientais para a aproximação entre a ciência e os instrumentos de política ambiental, como o licenciamento ambiental e a AIA

\section{Agradecimentos}

O presente trabalho foi realizado com apoio da Coordenação de Aperfeiçoamento de Pessoal de Nível Superior - Brasil (CAPES) - Código de Financiamento 001.

\section{Referências}

Araújo, R. C. P. Análise da atitude dos turistas com relação ao desenvolvimento da energia eólica no litoral cearense, Brasil. Turismo \& Sociedade, 7(2), 308-329, 2014. doi: 10.5380/tes.v7i2.35298

Arnett, E. B.; Erickson, W. P.; Horn, J.; Kerns, J. Relationships between bats and wind turbines in Pennsylvania and West Virginia: an assessment of fatality search protocols, patterns of fatality, and behavioral interactions with wind turbines. Bat conservation international. Austin, 2005. Disponível em: https://tethys.pnnl.gov/sites/default/files/ publications/Arnett_et_al_2005.pdf

Arnett, E. B.; Brown, W. K.; Erickson, W. P.; Fiedler, J. K.; Hamilton, B. L.; Henry, T. H.; Jain, A.; Johnson, G. D.; Kerns, J.; Koford, R. R.; Nicholson, C. P.; O’Connell, T. J.; Piorkowski, M. D.; Tankersley, R. D.; Patterns of bat fatalities at wind energy facilities in North America. Journal of Wildlife Management, 72(1), 61-78, 2008. doi: $10.2193 / 2007-221$

Arnold, T. W.; Zink, R. M. Collision mortality has no discernible effect on population trends of North American birds. PLoS ONE, 6(9), 1-6, 2011. doi: 10.1371/journal. pone. 0024708

Associação Brasileira de Energia Eólica. Brasil chega a 13 $G W$ de capacidade instalada de energia eólica. São Paulo: ABEEólica, 2018. Disponível em: http://www.abeeolica. org.br/noticias/brasil-chega-a-13-gw-de-capacidade-instalada-de-energia-eolica/

Baban, S. M. J.; Parry, T. Developing and applying a GIS-assisted approach to locating wind farms in the UK. Renewable Energy, 24, 59-71, 2001. doi: 10.1016/S0960- 


\section{1(00)00169-5}

Baerwald, E.; Barclay, R. Geographic variation in activity and fatality of migratory bats at wind energy facilities. Journal of Mammalogy, 90(6), 1341-1349, 2009. doi: 10.1644/09-MAMM-S-104R.1

Bakker, R. H.; Pedersen, E.; van den Berg, G. P.; Stewart, R. E.; Lok, W.; Bouma, J. Impact of wind turbine sound on annoyance, self-reported sleep disturbance and psychological distress. Science of the Total Environment, 425, 42-51, 2012. doi: 10.1016/j.scitotenv.2012.03.005

Barrios, L.; Rodríguez, A. Behavioural and environmental correlates of soaring-bird mortality at on-shore wind turbines. Journal of Applied Ecology, 41, 72-81, 2004. doi: 10.1111/j.1365-2664.2004.00876.x

Baxter, W.; Ross, W. A.; Spaling, H. Improving the practice of cumulative effects assessment in Canada. Impact Assessment and Project Appraisal, 19(4), 253-262, 2001. doi: $10.3152 / 147154601781766916$

Bernard, E.; Paese, A.; Machado, R. B.; Aguiar, L. M. S. Blown in the wind: bats and wind farms in Brazil. Natureza e Conservação, 12(2), 106-111, 2014. doi: 10.1016/j. ncon.2014.08.005

Bernard, E.; Aguiar, L. M. S.; Machado, R. B. Discovering the Brazilian bat fauna: a task for two centuries? Mammal Review, 41(1), 23-39, 2011. doi: 10.1111/j. 1365-2907.2010.00164.x

Bevanger, K.; Bevanger, K.; Berntsen, F.; Clausen, S.; Dahl, E. L.; Flagstad, Ø.; Follestad, A.; Halley, D.; Hanssen, F.; Johnsen, L.; Kvaløy, P.; Lund-Hoel, P.; May, R.; Nygård, T.; Pedersen, H.C; Reitan, O.; Røskaft, E.; Steinheim, Y.; Stokke, B.; Vang, R. Pre-and post-construction studies of conflicts between birds and wind turbines in coastal Norway (BirdWind): report on findings 2007-2010. Trondheim: Norwegian Institute for Nature Research, 2010. (NINA Reports 620). Disponível em: http://www.nina.no/archive/ nina/PppBasePdf/rapport/2010/620.pdf

Birdlife International. Data zone. 2018. Disponível em: http://datazone.birdlife.org/site/search

Bishop, I. D.; Miller, D. R. Visual assessment of off-shore wind turbines: the influence of distance, contrast, move- ment and social variables. Renewable Energy, 32, 814-831, 2007. doi: 10.1016/j.renene.2006.03.009

Borioni, R.; Gallardo, A. L. C. F.; Sánchez, L. E. Advancing scoping practice in environmental impact assessment: an examination of the Brazilian federal system. Impact Assessment and Project Appraisal, 35(3), 200-213, 2017. doi: 10.1080/14615517.2016.1271535

Brannstrom, C.; Gorayeb, A.; Mendes, J. S.; Loureiro, C.; Meireles, A. J. A.; Silva, E.V.; Freitas, A. L. R.; Oliveira, R. F. Is Brazilian wind power development sustainable? Insights from a review of conflicts in Ceará state. Renewable and Sustainable Energy Reviews, 67, 62-71, 2017. doi: 10.1016/j.rser.2016.08.047

Brannstrom, C.; Gorayeb, A.; Souza, W. F.; Leite, N. S.; Chaves, L. O.; Guimarães, R.; Gê, D. R. F. Perspectivas geográficas nas transformações do litoral brasileiro pela energia eólica. Revista Brasileira de Geografia, 63(1), 0328, 2018. doi: 10.21579/issn.2526-0375_2018_n1_p3-28

Brasil. Ministério do Meio Ambiente. Áreas prioritárias - Caatinga. 2015. Disponível em: http://www.mma.gov. br/images/arquivo/80049/Areas\%20Prioritarias/Caatinga/ BIOMA\%20CAATINGA.pdf

Brasil. Ministério do Meio Ambiente. Serviço Florestal Brasileiro. Ceará: inventário florestal nacional - principais resultados. Brasília: MMA, 2016. Disponível em: http:// www.florestal.gov.br/publicacoes/713-relatorio-inventario-florestal-nacional-ce

Brasil. Ministério do Meio Ambiente. Áreas prioritárias mapa: mapa de áreas prioritárias. 2018a. Disponível em: http://www.mma.gov.br/destaques/item/476

Brasil. Ministério do Meio Ambiente. Consulta - Relatórios de UC. 2018b. Disponível em: http://www.mma.gov.br/ areas-protegidas/cadastro-nacional-de-ucs/consulta-gerar-relatorio-de-uc.html

Braunisch, V. Coppes, J.; Bächle, S.; Suchant, R. Underpinning the precautionary principle with evidence: a spatial concept for guiding wind power development in endangered species' habitats. Journal for Nature Conservation, 24, 31-40, 2015. doi: 10.1016/j.jnc.2015.01.003

Brook, B. W.; Bradshaw, C. J. A. Key role for nuclear energy 
in global biodiversity conservation. Conservation Biology, 29(3), 702-712, 2015. doi:10.1111/cobi.12433

Brown, K. B. Wind power in northeastern Brazil: local burdens, regional benefits and growing opposition. Climate and Development, 3(4), 344-360, 2011. doi: 10.1080/17565529.2011.628120

Cabinet Office. Professional Policy Making for the twenty first century. 1999. Disponível em: https://ntouk.files. wordpress.com/2015/06/professional-policy-making-for-the-21st-century-1999.pdf

Caithness Windfarm Information Forum. Summary of wind turbine accident data to 31 March 2018. 2018. Disponível em: http://www.caithnesswindfarms.co.uk/AccidentStatistics.htm

Canter, L.; Ross, B. A basic need for integration - bringing focus to the scoping process. Impact Assessment and Project Appraisal, 32(1), 21-22, 2014. doi: 10.1080/14615517.2013.872848

Canter, L. W.; Kamath, J. Questionnaire checklist for cumulative impacts. Environmental Impact Assessment Review, 15, 311-339, 1995. doi: 10.1016/0195-9255(95)00010-C

Carneiro, F. O. M.; Rocha, H. H. B.; Rocha, P. A. C. Investigation of possible societal risk associated with wind power generation systems. Renewable and Sustainable Energy Reviews, 19, 30-36, 2013. doi: 10.1016/j.rser.2012.11.006

Chanchitpricha, C.; Bond, A. Conceptualising the effectiveness of impact assessment processes. Environmental Impact Assessment Review, 43, 65-72, 2013. doi: 10.1016/j. eiar.2013.05.006

Clarke, A. Wind energy progress and potential. Energy Policy, 19(8), 742-755, 1991. doi: 10.1016/03014215(91)90044-O

Clarke, S. Balancing environmental and cultural impact against the strategic need for wind power. International Journal of Heritage Studies, 15(2-3), 175-191, 2009. doi: $10.1080 / 13527250902890688$

Cortner, H. J. Making science relevant to environmental policy. Environmental Science and Policy, 3(1), 21-30, 2000. doi: 10.1016/S1462-9011(99)00042-8
Dahl, E. L. Bevanger, K.; Nygard, T.; Roskaft, E.; Stokke, B.G. Reduced breeding success in white-tailed eagles at Smøla windfarm, western Norway, is caused by mortality and displacement. Biological Conservation, 145, 79-85, 2012. doi: 10.1016/j.biocon.2011.10.012

Dai, K.; Bergot, A.; Liang, C.; Xiang, W. N.; Huang, Z. Environmental issues associated with wind energy - a review. Renewable Energy, 75, 911-921, 2015. doi: 10.1016/j. renene.2014.10.074

Davis, J.; Davis, S. J. Noise pollution from wind turbines: living with amplitude modulation, lower frequency emissions and sleep deprivation. In: Proceedings International Meeting on Wind Turbine Noise, 2., Lyon, 2007. Disponível em: $<$ https://docs.wind-watch.org/davis-noisepollutionfromturbines.pdf.

Delicado, A.; Figueiredo, E.; Silva, L. Community perceptions of renewable energies in Portugal: Impacts on environment, landscape and local development. Energy Research and Social Science, 13, 84-93, 2016. doi: 10.1016/j. erss.2015.12.007

Devine-Wright, P.; Howes, Y. Disruption to place attachment and the protection of restorative environments: a wind energy case study. Journal of Environmental Psychology, 30, 271-280, 2010. doi: 10.1016/j.jenvp.2010.01.008

Dimitropoulos, A.; Kontoleon, A. Assessing the determinants of local acceptability of wind-farm investment : A choice experiment in the Greek Aegean Islands. Energy Policy, 37, 1842-1854, 2009. doi: 10.1016/j.enpol.2009.01.002

Drewitt, A. L.; Langston, R. H. W. Assessing the impacts of wind farms on birds. Ibis, 148, 29-42, 2006. doi: 10.1111/j. 1474-919X.2006.00516.x

Dunn, G.; Bos, J. J.; Brown, R. R. Mediating the science-policy interface: Insights from the urban water sector in Melbourne, Australia. Environmental Science and Policy, 82, 143-150, 2018. doi: 10.1016/j.envsci.2018.02.001

Fernandes-Ferreira, H.; Mendonça, S. V.; Albano, C.; Ferreira, F. S.; Alves, R. R. N. Hunting, use and conservation of birds in Northeast Brazil. Biodiversity and Conservation, 21, 221-244, 2012. doi: 10.1007/s10531-011-0179-9

Ferrer, M.; De Lucas, M.; Janss, G. F. E.; Casado, E.; 
Muñoz, A. R.; Bechard, M. J.; Calabuig, C. P. Weak relationship between risk assessment studies and recorded mortality in wind farms. Journal of Applied Ecology, 49, 38-46, 2012. doi: 10.1111/j.1365-2664.2011.02054.x

Fischer, J.; Lindenmayer, D. B. Landscape modification and habitat fragmentation: a synthesis. Global Ecology and Biogeography, 16, 265-280, 2007. doi: 10.1111/j. 1466-8238.2006.00287.x

Fischer, T. B. Theory and practice of strategic environmental assessment: towards a more systematic approach. London: Routledge, 2007.

Fokaides, P. A.; Miltiadous, I. C.; Neophytou, M. K. A.; Spyridou, L. P. Promotion of wind energy in isolated energy systems: the case of the Orites wind farm. Clean Technologies and Environmental Policy, 16, 477-488, 2014. doi:10.1007/s10098-013-0642-2

Fox, A. D.; Desholm, M.; Kahlert, J.; Kjaer, T.; Ib, C.; Petersen, K. Information needs to support environmental impact assessment of the effects of European marine offshore wind farms on birds. Ibis, 148, 129-144, 2006. doi: 10.1111/j.1474-919X.2006.00510.X

Frantál, B.; Kunc, J. Wind turbines in tourism landscapes: czech experience. Annals of Tourism Research, 38(2), 499-519, 2011. doi: 10.1016/j.annals.2010.10.007

Frantál, B.; Urbánková, R. Energy tourism: an emerging field of study. Current Issues in Tourism, 20(13), 13951412, 2017. doi: 10.1080/13683500.2014.987734

Fundação Cultural Palmares. Comunidades remanescentes de Quilombos (CRQ's). Brasília, 2018. Disponível em: http://www.palmares.gov.br/comunidades-remanescentes-de-quilombos-crqs

FUNAI - Fundação Nacional Do Índio. Shape. 2018. Disponível em: http://www.funai.gov.br/index.php/shape

Garthe, S.; Hüppop, O. Scaling possible adverse effects of marine wind farms on seabirds: developing and applying a vulnerability index. Journal of Applied Ecology, 41, 724-734, 2004. doi: 10.1111/j.0021-8901.2004.00918.x

Gil, A. C. Como elaborar projetos de pesquisa. 4.ed. São Paulo: Atlas, 2002.
Girão, W.; Albano, C.; Campos, A. A.; Pinto, T.; Carlos, C. J. Registros documentados de cinco novos trinta-réis (Charadriiformes: Sternidae) no estado do Ceará, nordeste do Brasil. Revista Brasileira de Ornitologia, 16(3), 252-255, 2008. Disponível em: http://www4.museu-goeldi.br/revistabrornito/revista/index.php/BJO/article/view/3411

Global Wind Energy Council. Global Wind Report: Annual Market Update 2016. Brussels, 2017. Disponível em: http:// gwec.net/publications/global-wind-report-2/global-wind-report-2016/

González, M. A; Ena, V. Cantabrian capercaillie signs disappeared after a wind farm construction. Chioglossa, 3, 65-74, 2011. Disponível em: http://buleria.unileon.es/ xmlui/handle/10612/1786

Gorayeb, A.; Brannstrom, C.; Meireles, A. J. A. Wind power gone bad: Critiquing wind power planning processes in northeastern Brazil. Energy Research \& Social Science, 40, 82-88, 2018. doi: 10.1016/j.erss.2017.11.027

Goyal, M. Repowering-next big thing in India. Renewable and Sustainable Energy Reviews, 14, 1400-1409, 2010. doi: 10.1016/j.rser.2010.01.002

Hayes, M. A. Bats killed in large numbers at United States wind energy facilities. BioScience, 63(12), 975-979, 2013. doi: 10.1525/bio.2013.63.12.10

Helldin, J. O.; Jung, J.; Neumann, W.; Olsson, M.; Skarin, A.; Widemo, F. The Impacts of wind power on terrestrial mammals: a synthesis - report 6510. Stockholm, Swedish Environmental Protection Agency, 2012. Disponível em: http://naturvardsverket.se/Documents/publikationer6400/978-91-620-6510-2.pdf

Holmes, J.; Clark, R. Enhancing the use of science in environmental policy-making and regulation. Environmental Science and Policy, 11(8), 702-711, 2008. doi: 10.1016/j. envsci.2008.08.004

Hunt, S. Making competition work in electricity. New York: John Wiley, 2002.

Hurtado, J. P.; Fernández, J.; Parrondo, J. L.; Blanco, E. Spanish method of visual impact evaluation in wind farms. Renewable and Sustainable Energy Reviews, 8, 483-491, 2004. doi: 10.1016/j.rser.2003.12.009 
ICMBIO - Instituto Chico Mendes de Conservação da Biodiversidade. Relatório anual de rotas e áreas de concentração de aves migratórias no Brasil. Rio de Janeiro: CEMAVE/ ICMBio, 2016a.

ICMBIO - Instituto Chico Mendes de Conservação da Biodiversidade. Livro vermelho da fauna brasileira ameaçada de Extinção: sumário executivo. 2016b. Disponível em: http:// www.icmbio.gov.br/portal/images/stories/comunicacao/ publicacoes/publicacoes-diversas/dcom_sumario_executivo_livro_vermelho_ed_2016.pdf

Instituto de Medicina Integral Professor Fernando Figueira. Assistência saúde - DSEI CEARÁ. 2015. Disponível em: http://www1.imip.org.br/imip/assistenciaesaude/saudeindigena/dseiceara.html

Instituto de Pesquisa e Estratégia Econômica do Ceará. Anuário estatístico do Ceará - 2016. Disponível em: https://www.google.com/url?sa=t\&rct=j\&q=\&esrc=s\&source $=$ web $\& \mathrm{~cd}=3 \& \mathrm{cad}=$ rja $\&$ uact $=8 \& \mathrm{ved}=0$ ahUKEwj-8Y22ur3bAhXRPpAKHZGNAw8QFghGMAI\&url=http $\% 3 \mathrm{~A} \% 2 \mathrm{~F} \% 2 \mathrm{Fwww} 2$.ipece.ce.gov.br\%2Fpublicacoes $\% 2$ Fanuario $\% 2$ Fanuario $2016 \% 2$ Fterritorio $\% 2$ Ftabelas\%2FTabela\%25201.2.9.xls\&usg=AOvVaw1UK1HnxBNxY9PgG4hITxEm

IPHAN - Instituto do Patrimônio Histórico e Artístico Nacional. Bens tombados e processos de tombamento em andamento - Ceará. 2017. Disponível em: http://portal. iphan.gov.br/uploads/ckfinder/arquivos/Ceara_bens_tombados_novembro_2017.pdf

IPHAN - Instituto do Patrimônio Histórico e Artístico Nacional. O IPHAN no Ceará. Brasília: IPHAN, 2018. Disponível em: http://portal.iphan.gov.br/ce/pagina/detalhes/1069

INCRA - Instituto Nacional de Colonização e Reforma Agrária. Index of /i3geo/geodados. 2018. Disponível em: http://acervofundiario.incra.gov.br/i3geo/geodados/

IAIA - International Association for Impact Assessment. Principles of environmental impact assessment, best practice. IAIA, 1999. Disponível em: http://scholar.google.com/ scholar?hl=en\&btnG=Search\&q=intitle:PRINCIPLES $+\mathrm{O}-$ F+ENVIRONMENTAL+IMPACT+ASSESSMENT+BEST+PRACTICE\#4

Jaskelevičius, B.; Užpelkiene, N. Research and assessment of wind turbine's noise in vydmantai. Journal of Environmental Engineering and Landscape Management, 16(2), 76-82, 2008. doi: 10.3846/1648-6897.2008.16.76-82

Josimovic, B.; Pucar, M. The Strategic environmental impact assessment of electric wind energy plants: case study 'Bavaniste' (Serbia). Renewable Energy, 35, 1509-1519, 2010. doi: 10.1016/j.renene.2009.12.005

Kaldellis, J. K.; Kavadias, K. A.; Paliatsos, A. G. Environmental impacts of wind energy applications: " myth or reality ?". Fresenius Environmental Bulletin, 12(4), 326-337, 2003.

Katsaprakakis, D. A. A Review of the environmental and human impacts from wind parks. A case study for the Prefecture of Lasithi, Crete. Renewable and Sustainable Energy Reviews, 16, 2850-2863, 2012. doi: 10.1016/j. rser.2012.02.041

Katsaprakakis, D. A.; Christakis, D. G. The Exploitation of electricity production projects from Renewable Energy Sources for the social and economic development of remote communities. the case of Greece: an example to avoid. Renewable and Sustainable Energy Reviews, 54, 341-349, 2016. doi: 10.1016/j.rser.2015.10.029

Keith, D. W.; Decarolis, J. F.; Denkenberger, D. C.; Lenschow, D. H.; Malyshev, S. L.; Pacala, S.; Rasch, P. J. The Influence of large-scale wind power on global climate. Proceedings of the National Academy of Sciences, 101(46), 16115-16120, 2004. doi: 10.1073/pnas.0406930101

Kelm, D. H.; Lenski, J.; Kelm, V.; Toelch, U.; Dziock, F. Seasonal bat activity in relation to distance to hedgerows in an agricultural landscape in central europe and implications for wind energy development. Acta Chiropterologica, 16(1), 65-73, 2014. doi: 10.3161/150811014X683273

Kunz, T. H.; Arnett, E. B.; Cooper, B. M.; Erickson, W. P.; Larkin, R. P.; Mabee, T.; Morrison, M. L.; Strickland, M. D.; Szewczak, J. M. Assessing impacts of wind energy development on nocturnally active birds and bats: a guidance document. Journal of Wildlife Management, 71(8), 2449-2486, 2007. doi: 10.2193/2007-270

Kuvlesky, W. P.; Brennan, L. A.; Morrison, M. L.; Boydston, K. K.; Ballard, B. M.; Bryant, F. C. Wind energy development and wildlife conservation: challenges and opportuni- 
ties. Journal of Wildlife Management, 71(8), 2487-2498, 2007. doi: 10.2193/2007-248

Ladenburg, J.; Lutzeyer, S. The Economics of visual disamenity reductions of offshore wind farms - review and suggestions from an emerging field. Renewable and Sustainable Energy Reviews, 16, 6793-6802, 2012. doi: 10.1016/j.rser.2012.08.017

Langbroek, M.; Vanclay, F. Learning from the social impacts associated with initiating a windfarm near the former island of Urk, The Netherlands. Impact Assessment and Project Appraisal, 30(3), 167-178, 2012. doi: 10.1080/14615517.2012.706943

Leung, D. Y. C.; Yang, Y. Wind energy development and its environmental impact: a review. Renewable and Sustainable Energy Reviews, 16, 1031-1039, 2012. doi: 10.1016/j. rser.2011.09.024

Lima, F. A.; Marquesan, F. F. S. Terras indígenas no Ceará: uma história de lutas e resistência. Revista brasileira de estudos latino-americano, 7(3), 488-509, 2017. Disponível em: http://ojs.sites.ufsc.br/index.php/rebela/article/view/2561

Lima, M. C. Pesca artesanal, carcinicultura e geração de energia eólica na zona costeira do Ceará. Terra Livre, 2(31), 203-213, 2008. Disponível em: http://www.agb.org.br/ publicacoes/index.php/terralivre/article/view/270

Lopucki, R.; Mróz, I. An Assessment of non-volant terrestrial vertebrates response to wind farms - a study of small mammals. Environmental Monitoring and Assessment, 188, 122, 1-9, 2016. doi: 10.1007/s10661-016-5095-8

Lothian, A. Scenic perceptions of the visual effects of wind farms on South Australian landscapes. Geographical Research, 46(2), 196-2017, 2008. doi: 10.1111/j. 1745-5871.2008.00510.x

Loureiro, C. V.; Gorayeb, A.; Brannstrom, C. Implantação de energia eólica e estimativa das perdas ambientais em um setor do litoral oeste do Ceará, Brasil. Geosaberes, 6(1), 24-38, 2015. Disponível em: https://dialnet.unirioja.es/ servlet/articulo? codigo $=5548046$

Lovich, J. E.; Ennen, J. R. Assessing the state of knowledge of utility-scale wind energy development and operation on non-volant terrestrial and marine wildlife. Applied Energy,
103, 52-60, 2013. doi: 10.1016/j.apenergy.2012.10.001

Markevičius, A.; Katinas, V.; Marčiukaitis, M. Wind energy development policy and prospects in Lithuania. Energy Policy, 35(10), 4893-4901, 2007. doi: 10.1016/j. enpol.2007.03.026

Masden, E. A.; Fox, A. D.; Furness, R. W.; Bullman, R.; Haydon, D. T. Cumulative impact assessments and bird/ wind farm interactions: developing a conceptual framework. Environmental Impact Assessment Review, 30, 1-7, 2010. doi: 10.1016/j.eiar.2009.05.002

Mcgrath, C.; Bond, A. The Quality of environmental impact statements: a review of those submitted in Cork, Eire from 1988-1993. Project Appraisal, 12(1), 43-52, 1997. doi: 10.1080/02688867.1997.9727037

Meireles, A. J. A. Danos socioambientais originados pelas usinas eólicas nos campos de dunas do Nordeste brasileiro e critérios para definição de alternativas locacionais. Revista Franco-Brasilera de Geografia, 11, 1-25, 2011. Disponível em: https://journals.openedition.org/confins/6970

Mirasgedis, S.; Tourkolias, C.; Tzovla, E.; Diakoulaki, D. Valuing the visual impact of wind farms: an application in South Evia, Greece. Renewable and Sustainable Energy Reviews, 39, 296-311, 2014. doi: 10.1016/j.rser.2014.07.100

Molina-Ruiz, J.; Serrano, L. T. Identificación de impactos ambientales significativos en la implantación de parques eólicos: un ejemplo en el municipio de Jumilla (Murcia). Investigaciones Geográficas, 41, 145-154, 2006. doi: 10.1016/j.renene.2010.08.041

Morgan, R. K. Environmental impact assessment: the state of the art. Impact Assessment and Project Appraisal, 30(1), 5-14, 2012. doi: 10.1080/14615517.2012.661557

Morrison-Saunders, A. Principles for effective Impact Assessment: examples from western Australia. In: IAIA11 Conference Proceedings. Puebla, 2011. Disponível em: http://researchrepository.murdoch.edu.au/id/eprint/6631

Moura-Fé, M. M.; Pinheiro, M. V. A. Os Parques eólicos na zona costeira do ceará e os impactos ambientais associados. Revista Geonorte, 9(1), 22-41, 2013. Disponível em: http:// periodicos.ufam.edu.br/revista-geonorte/article/view/1142 Noguera, J. C.; Pérez, I.; Mínguez, E. Impact of terrestrial 
wind farms on diurnal raptors: developing a spatial vulnerability index and potential vulnerability maps. Ardeola, 57(1), 41-53, 2010.

Orloff, S.; Flannery, A. Wind turbine effects on avian activity, habitat use, and mortality in altamont pass and solano count wind resource areas: 1989-1991. Final report. Tiburon: California Energy Commission, 1992. Disponível em: https://nationalwind.org/wp-content/uploads/2013/05/ Orloff-and-Flannery-1992.pdf

Ortegon, K.; Nies, L. F.; Sutherland, J. W. Preparing for end of service life of wind turbines. Journal of Cleaner Production, 39, 191-199, 2013. doi: 10.1016/j.jclepro.2012.08.022

Ouammi, A.; Ghigliotti, V.; Robba, M.; Mimet, A.; Sacile, R. A Decision support system for the optimal exploitation of wind energy on regional scale. Renewable Energy, 37, 299-309, 2012. doi: 10.1016/j.renene.2011.06.027

Paglia, A. P. Fonseca, G. A. B.; Rylands, A. B.; Herrmann, G.; Aguiar, L. M. S.; Chiarello, A. G.; Leite, Y. L. R.; Costa, L. P.; Siciliano, S.; Kierulff, M. C. M.; Mendes, S. L.; Tavares, V. C.; Mittermeier, R. A.; Patton, J. L. Lista anotada dos mamíferos do Brasil. 2.ed. Occasional Papers in Conservation Biology. Conservation International, 6, 76, 2012. Disponível em: http://www.conservation.org/global/ brasil/publicacoes/Documents/annotated_checklist_of_brazilian_mammals_2nd_edition.pdf

Pedersen, E. Health aspects associated with wind turbine noise - results from three field studies. Noise Control Engineering Journal, 59(1), 47-53, 2011. doi: 10.3397/1.3533898

Pedersen, E.; Waye, K. P. Exploring perception and annoyance due to wind turbine noise in dissimilar living environments. In: Proceedings European Conference on Noise Control: advanced solutions for noise control, 6. Tampere, 2006. doi: 10.1121/1.1815091

Peterson, K. Quality of environmental impact statements and variability of scrutiny by reviewers. Environmental Impact Assessment Review, 30, 169-176, 2010. doi: 10.1016/j. eiar.2009.08.009

Petrova, M. A. NIMBYism revisited: public acceptance of wind energy in the United States. Wiley Interdisciplinary Reviews: Climate Change, 4, 575-601, 2013. doi: 10.1002/ wcc. 250
Piacentini, V. Q.; Aleixo, A.; Agne, C. E.; Maurício, G. N.; Pacheco, J. F.; Bravo, G. A.; Brito, G. R. R.; Naka, L. N.; Olmos, F.; Posso, S.; Silveira, L. F.; Betini, G. S.; Carrano, E.; Franz, I.; Lees, A. C.; Lima, L. M.; Pioli, D.; Schunck, F.; Amaral, F. R.; Bencke, G. A.; Cohn-Haft, M.; Figueiredo, L. F. A.; Straube, F. C.; Cesar E. Annotated checklist of the birds of Brazil by the Brazilian Ornithological Records Committee / Lista comentada das aves do Brasil pelo Comitê Brasileiro de Registros Ornitológicos. Revista Brasileira de Ornitologia, 23(2), 91-298, 2015. Disponível em: urn:lsid:zoobank.org:pub:30856542-FFD1-44CA-B249-9F321CD4CF4C

Punch, J.; James, R.; Pabst, D. Wind turbine noise: what audiologists should know. Audiology today, 20-32, 2010. Disponível em: https://www.windturbinesyndrome.com/ img/WindTurbineNoise.pdf

Rabin, L. A.; Coss, R. G.; Owings, D. H. The Effects of wind turbines on antipredator behavior in California ground squirrels (Spermophilus beecheyi). Biological Conservation, 131, 410-420, 2006. doi: 10.1016/j.biocon.2006.02.016

Rockström, J.; Steffen, W.; Noone, K.; Persson, A.; Chapin, F. S.; Lambin, E.; Lenton, T. M.; Scheffer, M.; Folke, C.; Schellnhuber, H.J.; Nykvist, B.; Wit, C. A.; Hughes, T.; Leeuw, S.; Rodhe, H.; Sörlin, S.; Snyder, P. K.; Costanza, R.; Svedin, U.; Falkenmark, M.; Karlberg, L.; Corell, R. W.; Fabry, V. J.; Hansen, J.; Walker, B.; Liverman, D.; Richardson, K.; Crutzen, P.; Foley, J. Planetary boundaries: exploring the safe operating space for humanity. Ecology and Society, 14(2), 32-63, 2009. doi: 10.1007/s13398-0140173-7.2

Rose, D. C. The case for policy-relevant conservation science. Conservation Biology, 29(3), 748-754, 2015. doi: 10.1111/cobi.12444

Roy, S. B. Simulating impacts of wind farms on local hydrometeorology. Journal of Wind Engineering and Industrial Aerodynamics, 99, 491-498, 2011. doi: 10.1016/j. jweia.2010.12.013

Roy, S. B.; Traiteur, J. J. Impacts of wind farms on surface air temperatures. Proceedings of the National Academy of Sciences, 107(42), 17899-17904, 2010. doi: 10.1073/ pnas. 1000493107 
Rydell, J.; Bach, L.; Dubourg-Savage, M. J.; Green, M.; Rodrigues, L.; Hedenström, A. Mortality of bats at wind turbines links to nocturnal insect migration?. European Journal of Wildlife Research, 56(6), 823-827, 2010. doi: 10.1007/s10344-010-0444-3

Saidur, R.; Rahim, N. A.; Islam, M. R.; Solangi, K. H. Environmental impact of wind energy. Renewable and Sustainable Energy Reviews, 15(5), 2423-2430, 2011. doi: 10.1016/j.rser.2011.02.024

Sánchez, L. E. Avaliação de impacto ambiental: conceitos e métodos. São Paulo: Oficina de Textos, 2008.

Santangeli, A.; Toivonen, T.; Pouzols, F. M.; Pogson, M.; Hastings, A.; Smith, P.; Moilanen, A. Global change synergies and trade-offs between renewable energy and biodiversity. GCB Bioenergy, 8(5), 941-951, 2016. doi: 10.1111/gcbb.12299

Santos, M.; Bastos, R.; Travassos, P.; Bessa, R.; Repas, M.; Cabral, J. A. Predicting the trends of vertebrate species richness as a response to wind farms installation in mountain ecosystems of northwest Portugal. Ecological Indicators, 10(2), 192-205, 2010. doi: 10.1016/j.ecolind.2009.04.014

Somenzari, M.; Amaral, P. P.; Cueto, V. R.; Guaraldo, A. C.; Jahn, A. E.; Lima, D. M.; Lima, P. C.; Lugarini, C.; Machado, C. G.; Martinez, J.; Nascimento, J. L. X.; Pacheco, J. F.; Paludo, D.; Prestes, N. P.; Serafini, P. P.; Silveira, L. F.; Sousa, A. E. B. A.; Sousa, N. A.; Souza, M. A.; Telino-Júnior, W. R.; Whitney, B. M. An Overview of migratory birds in Brazil. Papéis Avulsos de Zoologia, 58, 1-66, 2018. doi: 10.11606/1807-0205/2018.58.03

Steele, A. An Environmental impact assessment of the proposal to build a wind farm at Langdon Common in the North Pennines, UK. The Environmentalist, 11(3), 195-212, 1991. doi: 10.1007/BF01263233

Stewart, G.; Pullin, A.; Coles, C. Poor evidence-base for assessment of windfarm impacts on birds. Environmental Conservation, 34, 1-11, 2007. doi: 10.1017/ S0376892907003554

Tabassum-Abassi; Premalatha, M.; Abbasi, T.; Abbasi, S.A. Wind energy: increasing deployment, rising environmental concerns. Renewable and Sustainable Energy Reviews, 31 , 270-288, 2014. doi:10.1016/j.rser.2013.11.019
Thayer, R. L.; Freeman, C. M. Altamont: public perceptions of a wind energy landscape. Landscape and Urban Planning, 14, 379-398, 1987. doi: 10.1016/0169-2046(87)90051-X

Thygesen, J.; Agarwal, A. Key criteria for sustainable wind energy planning - lessons from an institutional perspective on the impact assessment literature. Renewable and Sustainable Energy Reviews, 39, 1012-1023, 2014. doi: 10.1016/j. rser.2014.07.173

Travassos, P.; Costa, H. M.; Saraiva, T.; Tomé, R.; Armelin, M.; Ramírez, F. I.; Neves. J. A Energia eólica e a conservação da avifauna em Portugal. SPEA, Lisboa: 2005. Disponível em: http://www.spea.pt/fotos/editor2/ aenergiaeolicaemportugal.pdf

Tsoutsos, T.; Tsouchlaraki, A.; Tsiropoulos, M.; Serpetsidakis, M. Visual impact evaluation of a wind park in a Greek island. Applied Energy, 86, 546-553, 2009. doi: 10.1016/j. apenergy.2008.08.013

Voigt, C. C.; Popa-lisseanu, A. G.; Niermann, I.; Kramer-schadt, S. The catchment area of wind farms for european bats: a plea for international regulations. Biological Conservation, 153, 80-86, 2012. doi: 10.1016/j. biocon.2012.04.027

Walsh-Thomas, J. M.; Cervone, G.; Agouris, P.; Manca, G. Further evidence of impacts of large-scale wind farms on land surface temperature. Renewable and Sustainable Energy Reviews, 16, 8, 6432-6437, 2012. doi: 10.1016/j. rser.2012.07.004

Wang, S.; Wang, S. Impacts of wind energy on environment: a review. Renewable and Sustainable Energy Reviews, 49, 437-443, 2015. doi: 10.1016/j.rser.2015.04.137

Wilson, J. C.; Elliott, M.; Cutts, N. D.; Mander, L.; Mendão, V.; Perez-Dominguez, R.; Phelps, A. Coastal and offshore wind energy generation: Is it environmentally benign? Energies, 3, 1383-1422, 2010. doi: 10.3390/en3071383

Wynne, B. Uncertainty and environmental learning. Reconceiving science and policy in the preventive paradigm. Global Environmental Change, 2(2), 111-127, 1992. doi: 10.1016/0959-3780(92)90017-2

Zamot, H. R.; O’neill-Carrillo, E.; Irizarry-Rivera, A. 
Analysis of wind projects considering public perception and environmental impact. In: Proceedings Annual North American Power Symposium, 37, 2005. doi: 10.1109/ NAPS.2005.1560594

Zhou, L.; Tian, Y.; Roy, S. B.; Thorncroft, C.; Bosart, L. F.; $\mathrm{Hu}, \mathrm{Y}$. Impacts of wind farms on land surface temperature. Nature Climate Change, 2(7), 539-543, 2012. doi: 10.1038/ nclimate 1505
Zwart, M. C.; Robson, P.; Rankin, S.; Whittingham, M. J.; McGowan, P. J. K. Using environmental impact assessment and post-construction monitoring data to inform wind energy developments. Ecosphere, 6(2), 1-11, 2015. doi: 10.1890/ES14-00331.1 